\title{
Adaptively Selecting Working Frequency to Reduce Disturbances in High Frequency Radar
}

\author{
R.Z. GuI* \\ The Key Laboratory of Embedded System and Service Computing \\ Ministry of Education College of Electronics \& Information Engineering \\ State Key Laboratory of Millimeter Waves, Tongji University, Shanghai 200092, China
}

\begin{abstract}
In high frequency radar, we should avoid noise disturbances in the radar's working-frequency segment. Moreover, the sidelobes of strong targets interfere with the detection of weak targets. A new method based on an adaptive selecting working-frequency is proposed. Frequency spectrum monitor is designed for selecting quiet frequency segment for the radar. Frequency spectrum monitor and the receiver of the radar are arranged to work according to special time periods respectively. So the radar can work in the frequency segments with lower noise disturbances. Moreover, there is no correlation between the noise and the useful echo signal, though the correlation between noises over very short time periods is strong, the noise data produced by frequency spectrum monitor can be exploited effectively. Adjusting system parameters in real-time by adaptive methods can be utilized to reduce noise disturbances. Algorithm based on the properties of crosscorrelation between noise and target is exploited for suppressing sidelobe disturbances of strong targets. Lastly, the feasibility of the methods is verified by processing actual radar data.
\end{abstract}

PACS: $84.40 . \mathrm{Xb}$

\section{Introduction}

High frequency $(\mathrm{HF})$ radar can measure ocean parameters, such as the surface current, surface wind and wave characteristics, over the horizon, making it a valuable tool for oceanography. It has a working frequency located in the range from $3 \mathrm{MHz}$ up to $30 \mathrm{MHz}$. In this frequency band, there are many disturbances. Moreover, the disturbances are time-varying. How to accurately remove these disturbances is an important research subject.

At present, there are two methods used to reduce noise. The first method involves improving antenna design and increasing frequency spectrum monitor (FSM) hardware which can be used to select a suitable low-disturbance frequency segment that can prevent noise from entering the receiver [1-4]. The second method, aimed at the noise in the echo wave, uses signal processing algorithms to reduce sensitivity to disturbance. In fact, noise still exists in the frequency segment in which the radar operates, though improving antenna design and increasing FSM hardware can reduce noise in the source signal $[5,6]$. Moreover, antenna improvements cannot prevent strong targets from interfering with weak targets [7, 8]. After considering the system of $\mathrm{HF}$ ground wave radar made by Radio Propagation Lab in Wuhan University based on the FSM technique, this paper proposes adaptive methods of restraining noise that exploit the information in the noise signal acquired by FSM hardware. The method

\footnotetext{
* e-mail: rzgui@tongji.edu.cn
}

can adjust system parameters in real-time to produce optimal noise reduction.

This paper is organized as follows. In the next section, we describe the time-varying noise. Then we illuminate how to acquire noise information by adjusting the working time of FSM hardware. In Sect. 3, we describe the full algorithm, including why we selected a recursive least squares (RLS) filter, what its function is, and why we chose a fast transversal filter (FTF). Section 4 contains the results of computer simulation demonstrating the properties of the new algorithm. Section 5 concludes the paper.

\section{Time-varying disturbances}

The disturbances faced in detection target with high frequency surface wave radar (HFSWR) include radar system noise, ocean clutter echo, island echo, zero frequency interference, thunder and meteor interference, ionosphere interference, radio communication interference, the other clutter interference and atmosphere noise interference [9-12]. Because the exterior disturbances are serious, radar system noise is not considered in the paper. The ocean clutter echo is produced through the interaction between high frequency electronic wave and the ocean wave whose wavelength is half of radar wavelength. The first order echo indicates the information of ocean surface current, the second order echo expresses the height of ocean wave which is caused by wind and wave field. The ocean clutter echo which expresses the information of ocean dynamic coefficients is ceaselessly variable, because the ocean surface current and ocean 
wave height are changed ceaselessly. The island echo is similar with the target echo except its Doppler frequency is zero. The information of island echo can be seen in different distance cells because of its big size. The detection of target with low velocity is disturbed by island and land echo $[13,14]$. Zero frequency interference is the disturbance locating in the zero frequency among Doppler frequency spectrum nearby. It is caused by the frequency synthesizer in radar. The base which is nearby zero frequency in the Doppler frequency spectrum caused by zero frequency interference is driven up. So the zero frequency interference disturbs the target detection intensely. The radio frequency interferences are made by lots of shortwave communication stations and broadcasts which are distributed in the high frequency segment which radar works in. Except the disturbances mentioned above, there are many other clutters and disturbances unknown the caused reason in the radar echo. Those clutters and disturbances distribute more widely in the distance and the Doppler frequency spectrum. The atmosphere disturbance constructs the background noise of ocean echo spectrum mainly. For the action of wind, the atmosphere disturbance commonly is nonstationary stochastic process, moreover its distribution does not satisfy the Gauss distribution. So many disturbances such as atmosphere noise disturbance, radio frequency disturbance, thunder and meteor interference are time-varying $[15,16]$.

We select the actual data which comes from the Radio Propagation Lab in Wuhan University to extract ocean dynamic parameters at 15:45 on April 132004 on the Zhujiajian Island of Zhejiang province in China. The radar parameters are set as follows. Carrying frequency is $7.958 \mathrm{MHz}$ and range resolution is $2.5 \mathrm{~km}$. Sweep period is $0.653 \mathrm{~s}$ and coherent integration time is about $13 \mathrm{~min}$. The disturbances faced in detection target are shown in Fig. 1.

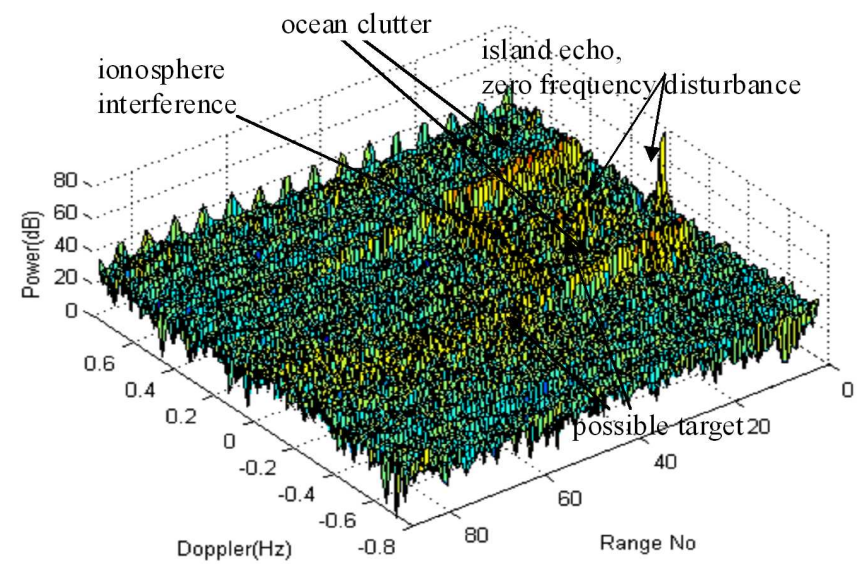

Fig. 1. Three dimensions figure of the radar actual data.

The disturbances such as ocean clutter, island echo, zero frequency disturbance, possible target are shown in
Fig. 1. Ocean clutter in the near distance cells is stronger that in the far distance cells. The background of far distance cells are weak obviously, and the all kinds of spike disturbances exist all distance cells. The distributions of those disturbances are nonhomogeneous in all distance cells. There are many different disturbances which occupy the zone of nonzero frequency, when the circumstance becomes atrocious. We deal with the time-varying circumstance of radar detection with the proposal which is to detect target.

\section{Working time of frequency monitor}

In this section, we proceed to show how to generate noise sample through FMS. Firstly, we control the hardware of FMS to scan the whole range in which HF ground wave radar can run effectively. Secondly, we choose some low-disturbance frequency segments according to the output of the first step. Then, we force the transmitter and the receiver to work in these frequency segments. Having finished the work of selecting frequencies, we change the scanning range of FSM so that only the selected frequency segments are scanned. Thus the working time of FSM hardware is shown in Fig. 2.

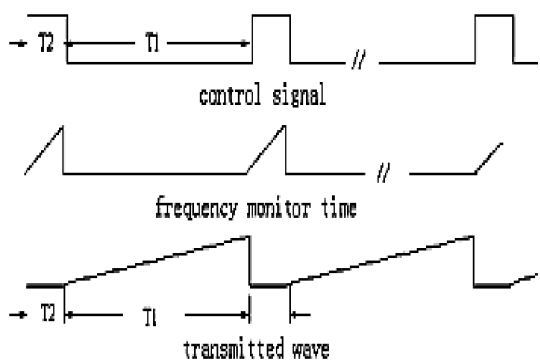

Fig. 2. Frequency spectrum monitor time.

Each period is divided into two parts T1, T2. T1 is the duration of radar's transmission of frequency modulated interrupting continuous wave (FMICW), we wake up the FSM in T2. In the period of T2, the work of FSM is not in the whole range as the initial operation of radar is. It just scans the frequency segments in which radar is working. Control signal is used to realize the switch between T1 and T2. Frequency monitor time shows that FSM works in the T2 period. The transmission of wave is finished in the radar working time $\mathrm{T} 1$. There exists one period in which the FSM hardware scans the frequency segments in which radar works. Doing so has two functions. One function is to provide noise sample for adaptive algorithm. The other is to monitor in real-time the noise that exists in these frequency segments in which radar works. If there is serious noise disturbance, the radar should be stopped. Then we repeat the work in the first step. That is to force the FSM hardware to search the whole range in which $\mathrm{HF}$ radar can possibly work. After new suitable frequency segments are chosen, the radar can work normally again. 


\section{Adaptive arithmetic}

The basic structure of the adaptive algorithm is shown in Fig. 3. The echo signal includes FMICW signal scattered back and unrelated noise. The combined signal and noise, $s(n)+N_{1}(n)$, forms the "primary input" to the adaptive algorithm. The "reference input" receives a noise $N_{2}(n)$ which is uncorrelated with the signal but correlated with the noise $N_{1}(n)$ in some unknown way. The noise $N_{2}(n)$ which is output of FSM is filtered to produce an output, $y(n)$, that is a close replica of $N_{1}(n)$. This output is subtracted from the primary input $s(n)+N_{1}(n)$ to produce the system output, $s(n)+N_{1}(n)-N_{2}(n)$. So $N_{1}(n)$ existing in echo signal can be suppressed in certain extent.

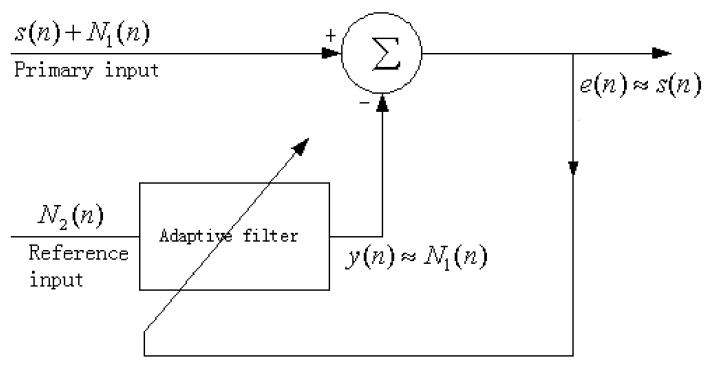

Fig. 3. Structure of adaptive algorithm.

Considering the disturbance by the strong target sidelobe, we can add the strong target signal which can be reconstructed through processing previous output of the baseband signal to the reference input. The influence of strong target sidelobe can be removed even completely by using the new adaptive algorithm. We selects transversal filter of FIR, because IIR is too complex to implement and there is the problem of stability. However, FIR can be easily implemented through transversal filter and lattice-type filter. At present, the least mean squares (LMS) and RLS are two different algorithms for implementation of adaptive filters. There are many differences such as those in principle, algorithm, and performance, between RLS and LMS, because they have discrepancy in optimal rule. LMS searches for the optimal filtering output according to the long-range statistical properties of input data. However, all we can do is to estimate the long-range statistical properties by using one group data. On the other hand, RLS is the recursive exponentially weighted least squares filtering algorithm. In RLS estimation, the main idea is to compute at each time $n$ for an optimal filter based on all the previous data. Furthermore, the parameter of filter at time $n$ is computed from data which is input at time $n$ as well as the parameter of filter at time $n-1$. Its quadratic cost function

$$
J(n)=\sum_{i}^{n} \lambda^{n-i}|\varepsilon(i)|^{2},
$$

where $\lambda$ weighted factor is called forget factor $(0<\lambda<$ 1). The memory is infinite, but "old" value is given a small weight so that it does not play a significant role.
Roughly speaking, only the latest data points are given large weights. To some extent, we can forget the noise data from FSM. That is, the latest noise data has the greatest influence on computing. The RLS algorithm is suitable for HF ground wave radar.

There is another factor which should be considered. Conventional RLS algorithm is applied in the case of additive white noise. However, the situation is complex in the case of frequency segment in which HF ground wave radar works. It could not be treated as white noise. Generally, the disturbance should be considered as colored noise. We can process the signal with conventional RLS algorithm after translating the colored noise into white noise. In fact, we can achieve the same effect with higher-order statistics (higher-order matrix, higher-order cumulant, and their spectra can be called higher-order statistics) to reconstruct conventional RLS code function. Higher-order statistics, particularly the higher-order cumulant, has many advantages such as its restraining Gauss noise, the phase information in it, its presenting the nonlinear properties of signal. In a word, this paper exploits fast transversal RLS based on higher-order cumulant.

Considering the complex and application of the computing operation, we choose the third order cost function, its expression is shown as follows. $x_{h}(n)$ represents the sample data of echo signal, $s(n)+N_{1}(n)$, which includes useful FMICW signal and noise. The sample data of the noise, $N_{2}(n)$, which is acquired through FSM, is represented by $x_{z}(n)$, third-order cost function is given by

$$
\begin{aligned}
J_{3} & =\sum_{k=1}^{n} s_{h}(k)\left[x_{h}(k)-\sum_{i=1}^{m} w_{i} x_{z}(k-i)\right]^{2} \\
& =\sum_{k=1}^{n} s_{h}(k)\left[x_{h}(k)-w^{\mathrm{T}} x_{z}(k)\right]^{2},
\end{aligned}
$$

where $s_{h}(k)=\sum_{m=-M}^{M} \beta(m) x_{h}(k-m), \quad w=$ $\left[w_{1}, \ldots, w_{m}\right]^{\mathrm{T}}$, and $\beta(m)$ is window function. The answer to third-order cost function is determined by the equation

$$
\left[\sum_{k=1}^{n} s_{h}(k) x_{z}(k) x_{z}^{\mathrm{T}}(k)\right] w=\sum_{k=1}^{n} s_{h}(k) x_{h}(k) x_{z}(k) .
$$

Using the knowledge of converse matrix and recursion, we can acquire the adaptive algorithm of RLS based on third-order cumulant as follows.

Initialization

$$
P\left(N_{0}\right)=C^{-1}\left(N_{0}\right),
$$

Renovation

$$
\begin{aligned}
& k=N_{0}+1, N_{0}+1, \ldots \\
& s_{h}(n)=\sum_{i=-M}^{M} \beta(i) x_{h}(n+i),
\end{aligned}
$$




$$
\begin{aligned}
& K(n)=\frac{s_{h}(n) P(n-1) x_{z}(n)}{\lambda+s_{h}(n) x_{z}^{\mathrm{T}}(n) P(n-1) x_{z}(n)}, \\
& a(n)=x_{h}(n)-x_{z}^{\mathrm{T}}(n) w(n-1), \\
& w(n)=w(n-1)+K(n) a(n), \\
& P(n)=\lambda^{-1} P(n-1)-\lambda^{-1} K(n) x_{z}^{\mathrm{T}}(n) P(n-1) .
\end{aligned}
$$

RLS based on the fast transversal filter algorithm can make up for the disadvantage caused by using third-order cumulant. We can process the signal of radar in real-time.

\section{Simulating results}

In the section, computer simulation results will be examined to demonstrate the effectiveness of the presented algorithm. The following parameters of FMICW signal of HF radar are assumed. Carrier frequency $f_{\mathrm{c}}=6 \mathrm{MHz}$, bandwidth $B=40 \mathrm{kHz}$, time width $T=1 \mathrm{~s}$, the number of samples in each period $N=1024$, range resolution $\Delta R=3.75 \mathrm{~km}$. We simulate colored noise by means of letting white noise go through the net which has the function of frequency selection.

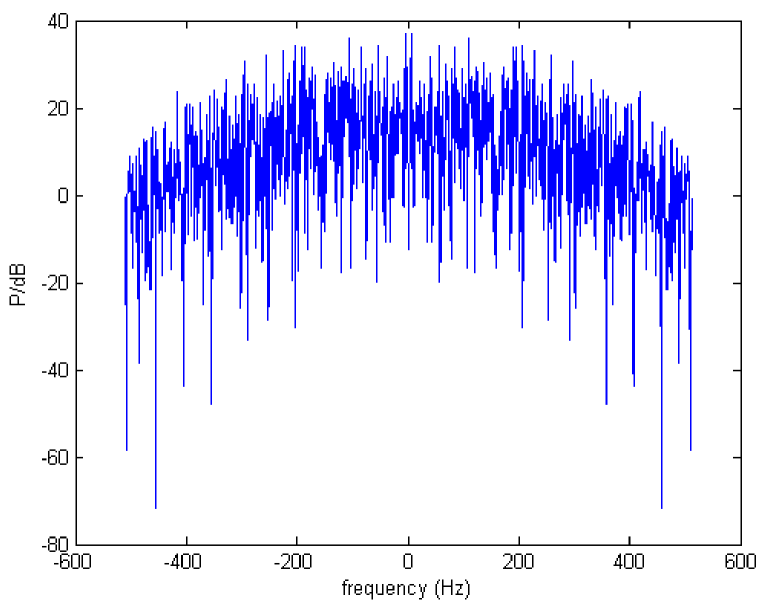

Fig. 4. Colored-noise spectrum.

Its spectrum is shown in Fig. 4. Four targets are located in positions of $153.75 \mathrm{~km}, 191.25 \mathrm{~km}, 228.75 \mathrm{~km}$, $266.25 \mathrm{~km}$, respectively. We use general FFT algorithm to process the sample of baseband signal.

The result of processing is shown in Fig. 5. The four target can be presented by four vertical lines whose frequencies are $41 \mathrm{~Hz}, 51 \mathrm{~Hz}, 61 \mathrm{~Hz}, 71 \mathrm{~Hz}$ according to the switch between distance and frequency in the double sides spectrum. Signal to noise ratios (SNR) is $18 \mathrm{~dB}$. We process the sample of baseband signal using adaptive algorithm based on RLS.

The result is shown in Fig. 6. The SNR is $37 \mathrm{~dB}$. It is obvious that the new adaptive algorithm can improve the SNR by reducing the power of noise but not changing the power of signal. We continue to simulate how to suppress the disturbances of sidelobes of strong targets that interfere with the detection of weak targets with the

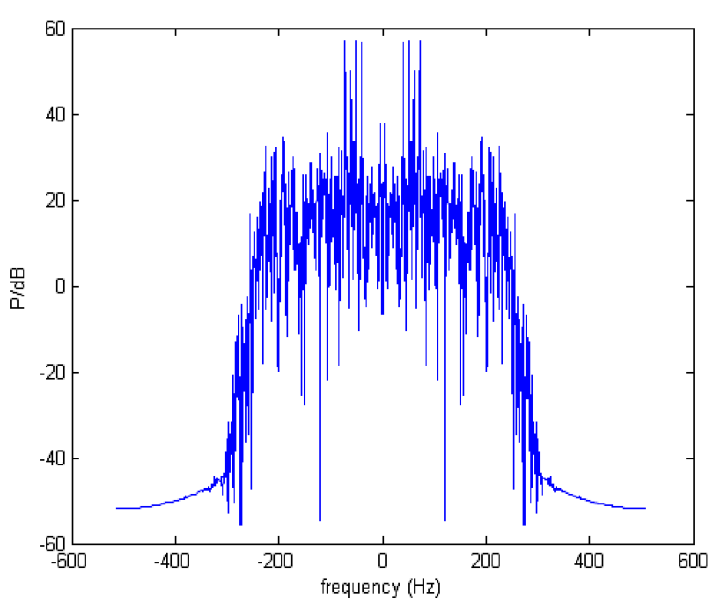

Fig. 5. Double sides spectrum using FFT.

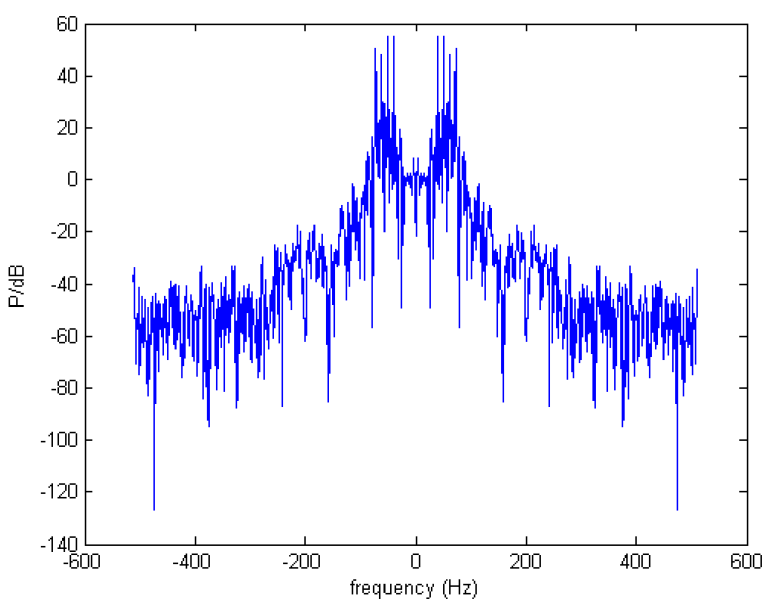

Fig. 6. Double sides spectrum using RLS.

new adaptive algorithm. The four targets are located in positions of $191.25 \mathrm{~km}, 195 \mathrm{~km}, 198.75 \mathrm{~km}$, and $202.5 \mathrm{~km}$. The power of the two middle targets is twice as strong as that of the others.

The result of processing of the sample of baseband signal with general FFT algorithm is shown by means of the single spectrum ranging from 0 to $200 \mathrm{~Hz}$ in Fig. 7. The two weak signals cannot be shown in Fig. 7. The strong target signal can be treated as noise when the weak target is detected. We add signals of two strong targets that can be reconstructed easily by processing the sample of baseband signal to the reference input of adaptive algorithm.

We can see distinctly the spectrum of the two weak targets located at $191.25 \mathrm{~km}, 202.5 \mathrm{~km}$ in Fig. 8 .

For verifying the feasibility of the methods mentioned above, we are processing the actual radar data with the methods. We select the actual data which comes from the Radio Propagation Lab in Wuhan University to extract ocean dynamic parameters at 15:45 on April 13, 2004 on the Zhujiajian Island of Zhejiang province in China. The 


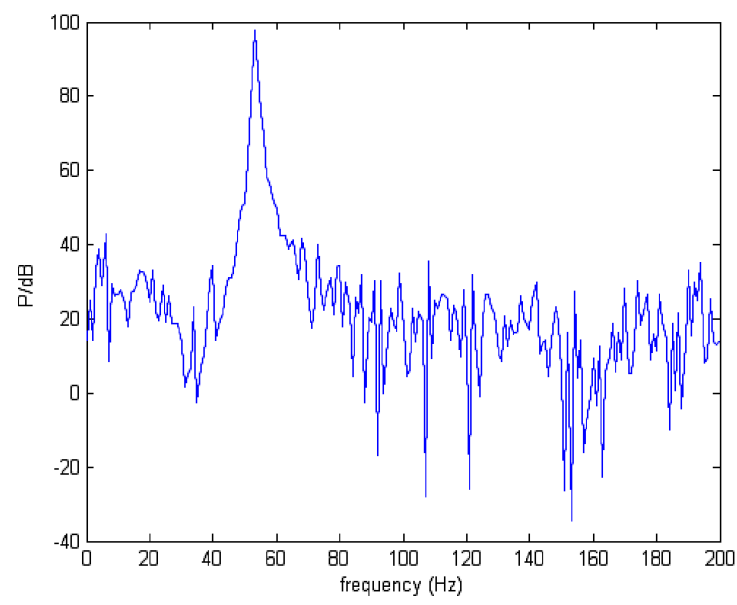

Fig. 7. Single side spectrum using general FFT.

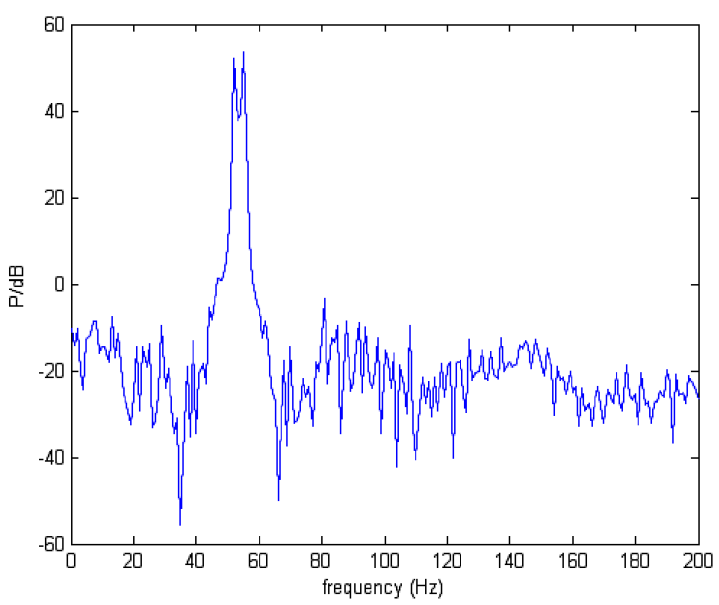

Fig. 8. Single side spectrum using RLS.

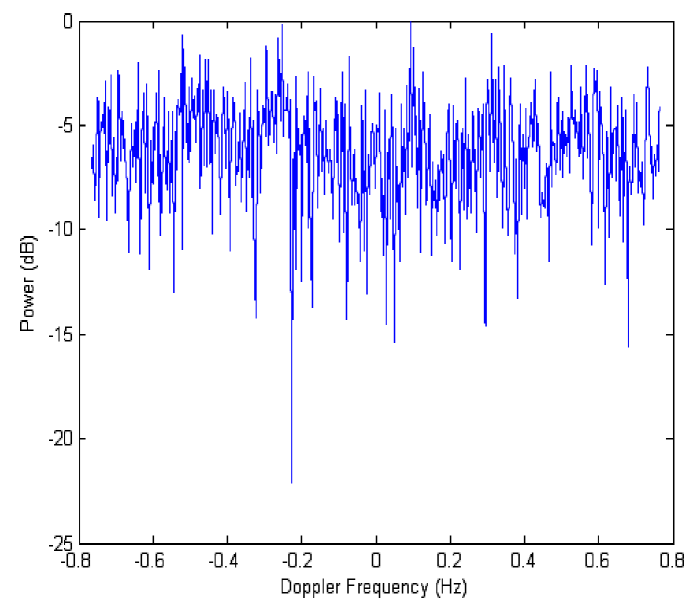

Fig. 9. Pre-processing actual radar data.

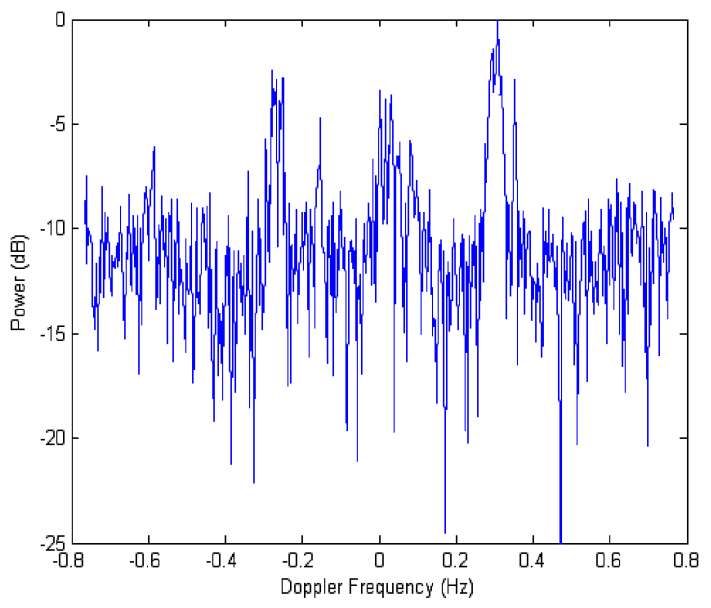

Fig. 10. Post-processing actual radar data.

radar parameters are set as follows. Carrying frequency is $7.958 \mathrm{MHz}$ and range resolution is $2.5 \mathrm{~km}$. Sweep period is $0.653 \mathrm{~s}$ and coherent integration time is about 13 min. The distance's cell is the 41th. The results of pre-processing and post processing with the method are shown in Fig. 9 and Fig. 10, respectively.

The signal-to-noise ratio is improved with the method. The radar cross-section is variable according to the kinds of vessel. The actual radar data about the detecting targets cannot be gotten easily. So it is difficult to verify the influence of the sidelobe of strong targets on the detection of weak targets.

\section{Conclusions}

The paper presented a new adaptive algorithm to deal with noise and sidelobe disturbances. The method of adaptive algorithm exploited the FSM hardware effectively while preserving the hardware of HF radar made by Radio Propagation Lab in Wuhan University. With reference to the changes of noise, we can realize the optimal restraint of disturbances in real-time. By replacing the general FTF algorithms in some complex system with algorithms of parallel fast adaptive RLS and KaGEt RLS, we can produce more rapid adaptive algorithm in future.

\section{Acknowledgments}

The author acknowledges the support from Open Research Fund of State Key Laboratory of Information Engineering in Surveying, Mapping and Remote Sensing 09R05 and State Key Laboratory of Millimeter Waves K200916, the Scientific Research Foundation for the Returned Overseas Chinese Scholars, State Education Ministry, and Rohde \& Schwarz GmbH Germany. 


\section{References}

[1] Li Hui, Yang Zijie, J. Wuhan Univ. (Natural Science Edition) 10, 653 (2003) (in Chinese).

[2] R.Z. Gui, Z.J. Yang, Radio Science 41, RS4S90 (2006).

[3] R.Z. Gui, IEEE Radar Symp., 213, Sept. 2009.

[4] R.Z. Gui, Tongji XueBao 36, 967 (2008) (in Chinese).

[5] B.S. Wei, Y.T. Liu, J. Electron. 3, 437 (2003) (in Chinese).

[6] F. Gini, M. Rangaswamy, Knowledge-Based Radar Detection, Tracking, and Classification, WileyInterscience, 2008, p. 103.

[7] D.T.L. Lee, B. Friedlander, IEEE Trans. ASSP-29, 467 (1981).

[8] G.B. Giannakis, J.M. Mendel, IEEE Trans. 37, 360 (1989).
[9] J.M. Cioffi, T. Kailath, IEEE Trans. ASSP-33, p. 607 (1985).

[10] K. Takeichi, T. Furukawa, in: ISCAS 2002. IEEE Int. Symp., 2002, Vol. 1, p. I-21.

[11] I.D. Skidmore, I.K. Proudler, IEEE Trans. 51, 3094 (2003).

[12] M. Yaremchuk, A. Sentchev, Continental Shelf Res. 29, 1711 (2009).

[13] E.W. Gill, J. Walsh, Radio Sci. 43, RS4 012 (2008).

[14] G.A. Fabrizio, A. Farina, M.D. Turley, IEEE Trans. Aerosp. Electron. Syst. 41, 922 (2005).

[15] J.M. Headrick, S.J. Anderson, in: Radar Handbook, Ed. M.I. Skolnik, 3rd ed., McGraw-Hill, New York 2008, Ch. 20, p. 1.

[16] H. Leong, A. Ponsford, in: Radar Conf. 2008, IEEE 2008 , p. 1 